\title{
Urban resilience in a context of climate change
}

\author{
Kamilia Mahdaoui* and Jamal Alibou \\ CEDoc EHTP, Civil, Hydraulic, Climate and Environmental Laboratory, KM7, El Jadida Road, B.P 8108, Casablanca, Morocco
}

\begin{abstract}
Morocco has experienced many weather events that have been increasingly aggravated by climate change, a global, irreversible and anthropogenic phenomenon. They have a major impact on the main sectors of the economy and on the infrastructure. The objective of the study is to propose adaptation options and the plan for their implementation to improve the resilience of certain infrastructure to climate risk, a necessary but not sufficient condition to ensure the smooth functioning of the city. The methodological approach used is the quantitative approach which consists in quantifying the selected indicators attributed to the key elements of the impact chains and normalizing, weighting and aggregating them in order to obtain as desired results the vulnerability and climate risk scores in the form of tables and interactive maps of the study area for a better interpretation (Very low, low, medium, high, very high). On the basis of these results, effective and operational adaptation measures will be suggested. Thus, having a plan for implementing adaptation options is vitally important for a city to be resilient and less vulnerable to climate risk.
\end{abstract}

\section{Introduction}

The novelty of climate change consists mainly in an intensification of natural hazards whose rates of occurrence are estimated as "highly probable" by global climate models leading to important repercussions on the major components of the city especially infrastructures which are critical because they are very important for the proper functioning of societies and vulnerable due to their exposure to hazards and their interdependence on each other.

As urban societies are highly dependent on the proper functioning of their infrastructures, their failure are often a primary cause of human and economic vulnerability. This is why improving the resilience of critical infrastructure is among the necessary conditions to improve the resilience of the city.

Currently, almost all Moroccan cities are expressing their desire to find answers to the impacts of climate change which implies the existence of real adaptation plans, effective and operational in their territories with the aim of improving urban resilience. Nevertheless, at present, the majority of these cities don't yet have any adaptation plan and among the cities that do, many only begin to face the challenges of implementing such a plan. The objective then is to mobilize to determine the appropriate adaptation measures in order to have strong and resilient infrastructures. The following questions need to be answered to solve the problematic:

- To which changing climate signals is the system exposed? And what characteristics make this system susceptible to changing climate conditions?

- How to select indicators for components of the impact chains? What kind of data do we need?
- Which methods can be used to normalize indicators, to define their weights and how should they be aggregated?

-How to combine several indicators into a composite indicator representing a vulnerability component and a climatic risk?

\section{Literature review}

A number of key concepts used must be defined within the theoretical framework, namely: city, critical infrastructure, vulnerability, climate risk, resilience, and adaptation.

The city appears both vulnerable and best positioned to deal with climate risk. The concept of the city has been defined differently by many researchers. For some, the city is an object constructed by our representations: "We grasp the city through our representations that means the mental images that emerge from our personality or culture, our desire to understand, our willingness to act. Thus, we see the city through what we are, but also through our way of thinking, through what we want. So, the representations of the city are multiple, as is the man who thinks it is multiple"(p.51).[1] For others, the city is seen as a system that is "a set of elements in dynamic interaction organized according to a purpose"(p.3).[2] more precisely, it will be seen as a socio-technical system where critical infrastructures occupy a central place "The city, which today is home to more than half of the world's inhabitants, is not only a form of densely populated area, which brings together a certain number of political, administrative and economic functions, but also a form of management of drinking water, wastewater, energy and waste issues"(p.2).[3] Without repeating all the existing literature on the city, we can say that the city is a

\footnotetext{
*Corresponding author: Kamilia-Mahdaoui@outlook.fr
} 
sociotechnical system in which critical infrastructures play a primordial role in its functioning.

Critical infrastructures are the subject of particular attention in the majority of countries as shown in the table 1(p.11)[4] which exposed some definitions of this term:

Table 1: Critical infrastructures - Some definitions

\begin{tabular}{|l|c|}
\hline \multicolumn{1}{|c|}{ Definition } & Source \\
\hline Critical infrastructure consist of those physical & \\
and information technology facilities, & \\
networks, services and assets which, if & EU \\
disrupted or destroyed, would have a serious & \\
impact on the health, safety and security. & \\
\hline $\begin{array}{l}\text { Critical infrastructures (CI) include } \\
\text { organizations and systems with tremendous }\end{array}$ & \\
importance for the society that, if disrupted, & Germany \\
would have sustained influence on supply & \\
chains and public safety and could lead to & \\
further dramatic consequences. & \\
\hline $\begin{array}{l}\text { Infrastructures are deemed critical if they } \\
\text { constitute an essential, indispensable facility }\end{array}$ & \\
for society, and if their disruption would \\
rapidly bring about a state of emergency or \\
could have adverse societal effects in the \\
longer term.
\end{tabular}

Therefore, critical infrastructure is defined as goods and services (networks, energy, health, etc.) that are of vital importance to the population and the economy. This is way most of countries are interested in them. There are two reasons for their criticality: The first reason is the impossibility for societies to deal with its dysfunction and the second is their interdependence on each other, meaning that the failure of one infrastructure will lead to the failure of another, thus leading to chain failures (Domino effect).

As for the concept of vulnerability, there are a variety of definitions in the available literature. In our study, we are interested in definitions of vulnerability in the scientific language of natural hazards and climate change.

They can be grouped into three groups:

The first one is related to physical sciences, we speak about technical vulnerability, and refers to the whole physical damage of a hazard on issues (infrastructures, buildings, populations, etc.).

The second is part of the humanities and social sciences, we talk about social vulnerability, it refers to the absence of society's capacity to face a crisis or change, the difficulty of a person, a group of people, an organization or a territory to anticipate a destructive phenomenon, to face it, to resist it and to recover after its occurrence (p.143) [5]. This type of vulnerability denounces, in a way, social fragility and social inequalities (income, age, etc.).

For the third group, it is the global vulnerability which brings together the two previous vulnerabilities.

So, the city's global vulnerability to climate change expresses at what point materials and humans issues can resist the hazard (According to their degree of sensitivity and exposure) and what a point the society is capable of coping and rebuilding itself (According to their intrinsic characteristics).

Since the critical infrastructures were considered key elements of the city, their vulnerability must then be questioned. The vulnerability of infrastructures is defined by the rate of damage to these infrastructures according to their sensitivity and exposure to hazards and also by the fragility factors contributing to the occurrence of damage in the event of a hazard.

The notion of vulnerability is associated with the notion of risk which the Intergovernmental Panel on Climate Change (IPCC) has defined as "The possible and uncertain consequences [ = impact or effect] of an event on something of value (...). Risk results from the interaction of vulnerability, exposure and hazards (...)" (p.5).[6] Climate risk then refers to climate events that have a particular impact on the territory, its inhabitants and its assets. They can take different forms: floods, cold waves and snowfall, heat waves, storms, rising sea levels, risk of erosion and loss of space...

Like vulnerability, resilience is a polysemic concept, subject to multiple definitions and specifications (see figure 1).

In physical science, resilience is the ability of a system to return to its initial state after a shock or continuous pressure, it is physical resilience (p.116).[7] For ecological resilience, it is defined as the capacity of an ecosystem to adapt to new situations (p.97).[8]

In psychology, resilience is the ability to live, succeed and develop in spite of adversity (p.1).[9]

In social science, Adger has defined social resilience as the capacity of human communities to support and recover from external shocks or disruptions. Most recently, he contributed to evolve the terminology in order to use the notion of eco-social resilience in the context of climate change. It concerns humans and nature as interdependent systems (p.6).[10]

In dynamic systems science, according to Dovers and Handmer (1992, 1996), systemic resilience takes two forms: the first is reactive resilience which presents the maximum of disturbance a system can undergo while remaining in the same state. It is directly linked to selforganization. The second is proactive resilience which is the system's ability to build and increase its adaptive capacity and learning capacity, which means its ability to 
recover from damage suffered by transforming, reorganizing or renewing the structures and functions of a system (p.101).[8]

What we can retain as a definition is that urban resilience corresponds to the city's ability to prepare and plan for the occurrence of hazards by anticipating response measures and its ability to function despite the disruption of some of its components (to operate in degraded mode), then to put them back into service so that they can return to optimal functioning as quickly as possible while taking into account the impacts endured with a view to minimize them during future disruptions. The same definition is applied to infrastructure resilience.

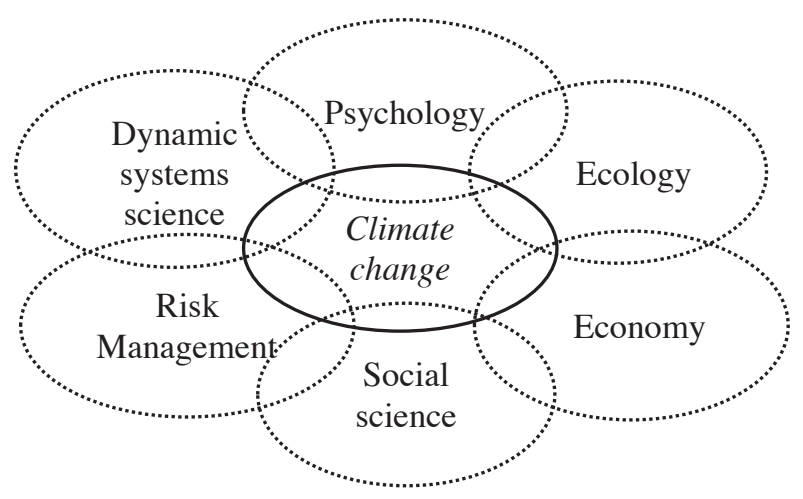

Fig. 1. Etymology of the term Resilience.

The use of the term adaptation is recent in the context of climate change. This can be explained by the fact that most of the research carried out has focused on hazard. The mobilization of this notion only began with the IPCC in the Third Assessment Report published in 2003 which, in chapter 18 entitled Adaptation to climate change in the context of sustainable development and equity [11], explains the concept in detail. The IPCC has given a universal definition of adaptation to climate change: "The process of adjusting to actual or expected climate and its effects. For human systems, it is about mitigating adverse effects and exploiting beneficial effects"(p.5).[6] Adaptation then consists in making systems less vulnerable to climate change, through actions that reduce the actual impacts of climate change or improve the response capacities of societies and the environment.

\section{Method}

As previously emphasized, the city is a system that maintains relations with its environment and interrelationships between its different components (population, infrastructure, businesses, etc.) characterized by a certain complexity. These relationships can be factors of vulnerability or resilience to the impacts of climate change. Consequently, a systemic vision applied to the urban domain appears indispensable.

The methodological approach to be followed is the quantitative approach because the objective is to propose a set of adaptation measures, prioritize them and develop a plan for their implementation. To do this, it is necessary to go through risk assessment. This operation requires the quantification of many elements such as: climatic factors, stress factors, sensitivity, adaptive capacity, vulnerability, impacts and risk by indicators that must be standardized, weighted and aggregated. The following diagram (see figure 2) (p.37) [12] explains the relationship between these elements, which is called the Impact Chain.

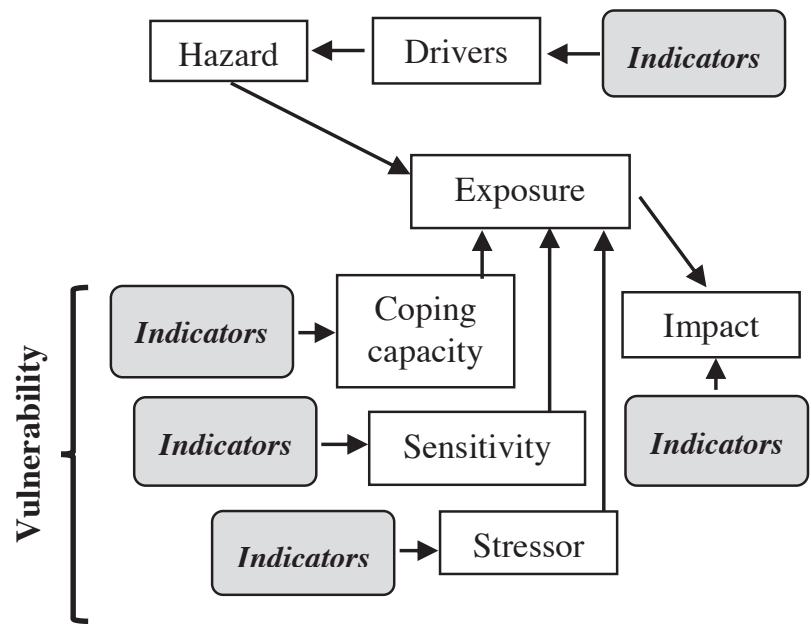

Fig. 2. Impact chain diagram scheme.

Hazard is defined as "[...] the potential occurrence of a natural or human-induced physical event or trend, or physical impact that may cause loss of life, injury, or other health impacts, as well as damage and loss to property, infrastructure, livelihoods, service provision, and environmental resources"(p.5).[6] The concept of effective exposure is the portion of the exposed assets that is actually affected by a specific hazard occurrence.

Drivers are climatic factors that causes those hazards.

Stressors are Non-climatic events that may have a major effect on the system exposed. For example, Population growth or percentage of sealed surface will increase the risk of inundation.

Sensitivity is defined by the degree to which a system could be affected by the considered hazard. Different systems are more or less sensitive to a hazard.

Coping capacity is the ability of people, institutions, organizations and systems to deal with, manage and overcome pejorative situations in the short to medium term using available skills, values, resources and opportunities.

Impact refers to undesired effects on natural, human and material systems like, livelihoods, health, services, infrastructure, ecosystems, economies, etc. of extreme weather and events due to climate change within a specific time period. Impacts are also called consequences and outcomes.

Vulnerability is then derived from the interplay of the elements stressors, sensitivity, and coping capacity. It contributes directly to the impact that a hazard causes to the exposed objects.

Indicators are employed to quantify the intensifying or mitigating elements of an exposed system with regard 
to selected hazard(s), as well as the potential impacts hazards may have on the exposed system. For instance, indicator socio-economic like population density, environmental indicator like change in average temperature or infrastructure indicator as water supply measured in number day, etc. The values of the selected indicators will later be aggregated to risk components and provide the basis for the calculation of the composite risk score (p. 47).[12]

Figure 3 shows an example of Impact chain diagram for Flood hazard on drinking water network.

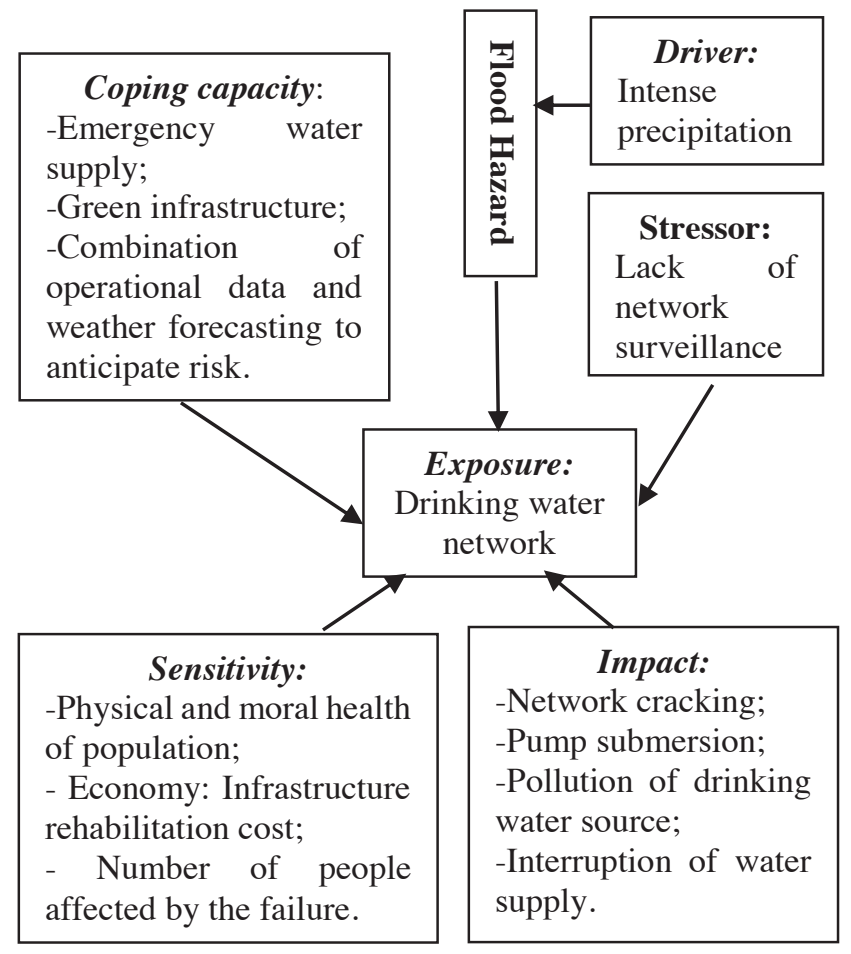

Fig. 3. Example of Impact chain diagram for Flood hazard on drinking water network.

For the procedure to follow, it consists of:

- Developing an impact chain which describes cause-effect relationship between elements (Qualitative stage);

- Identifying indicators to quantify the previously defined elements of impact chain diagrams;

- Acquiring and analyzing the necessary data sets or the measurement of the indicators which each in itself and in combination already may provide you with an impression of the vulnerability of the examined system;

- Normalizing indicators. In fact, the indicators selected and calculated use different measurement units and scales, they need to be normalized to be combined into risk components. Normalization is the process of using mathematical operations to transform values measured on different scales and in different units into unit-less values on a common scale. This operation allow to assess the criticality of different indicators values;
- Weighting and aggregating the coping capacity and sensitivity indicators to the risk component vulnerability. The indicators for the other risk components don't have to be aggregated any further;

- Estimating hazard intensity/probability and Impact. The probability value and the different (aggregated) impact values are used to determine the risk value;

- Presenting validated result;

- Determining and prioritizing adaptation options based on the results found;

- Defining an implementation plan for these options.

\section{Expected results}

The desired outcome after applying the methodology defined above is to obtain vulnerability and climate risk scores in the form of tables and interactive maps of the study area for a better interpretation (Very low, low, medium, high, very high).On the basis of these results, we will have a practical and effective implementation plan for adaptive measures appropriate to the climatic risk with starting points for action and obstacles need to be overcome in order to reduce risk effects and build resilience.

However, this approach didn't take into account the existing interdependence between the different infrastructures. Loss of electric power, for example, commonly leads to disruptions in water, transportation and health care systems, among others. The links between these infrastructures must not be neglected and should include in any study of urban resilience.

\section{Conclusion}

In conclusion, any city without an adaptation strategy will be considered behind the times or negligent in its responsibilities to protect its citizens, economy and quality of life.

\section{References}

1. S. Lhomme, Les réseaux techniques comme vecteur de propagation des risques en milieu urbain-Une contribution théorique et pratique à l'analyse de la résilience urbaine, p.365, (Janvier, 2013).

2. G. Donnadieu, D. Durand, D. Neel, E. Nunez, et L. Saint-Paul, App. Syst., p. 11.

3. S. Petitet, E.Ass.E.D. : V.V. ss R. ? p. 5.

4. J.-P. Galland, Crit. N.IC., 81, p. 18, (2010).

5. B. Quenault, Vuln., Cpt. C. An. RU. E.L.Avc .CC., p139-151, (2015).

6. GIEC, R. K. Pachauri, et L. A. Meyer, Changements climatiques 2014 : rapport de synthèse : contribution des Groupes de travail I, II et III au cinquième Rapport d'évaluation du Groupe d'experts 
intergouvernemental sur l'évolution du climat, (2015).

7. A. Dauphiné et D. Provitolo, Res. : Cpt. pr. GR., Ann. Géographie, 654, n² 2, p. 115, (2007).

8. B. Quenault, P. Pigeon, F. Bertrand, et N. Blond, Vulnérabilités et résilience au changement climatique en milieu urbain : vers de nouvelles stratégies de développement urbain durable? p. 204, (2011).

9. http://lionel.mesnard.free.fr/le $\% 20$ site/psyresilience.html

10. B. Barroca, M. DiNardo, et I. Mboumoua, De Vuln. à Res. : mut. ou boulvt. ?, EchoGéo, $n^{o}$ 24, (Juin 2013).

11. Troisième rapport d'évaluation du GIEC. https://library.wmo.int/index.php?lvl=bulletin_displ ay\&id=4027\#.X0_St31Kj6Q.

12. E. Rome, O. Ullrich, D. Lückerath, R. Worst, J. Xie, et M. Bogen, IVAVIA: Impact and Vulnerability Analysis of Vital Infrastructures and Built-Up Areas ", in Critical Information Infrastructures Security, 11260, E. Luiijf, I. Žutautaite, et B. M. Hämmerli, Éd. Cham: Springer International Publishing, p. 100, (2019). 